\title{
ORIGINAL ARTICLE \\ Purging deleterious mutations in conservation programmes: combining optimal contributions with inbred matings
}

\begin{abstract}
MÁR de Cara ${ }^{1}$, B Villanueva ${ }^{1}$, MÁ Toro ${ }^{2}$ and J Fernández ${ }^{1}$
Conservation programmes aim at minimising the loss of genetic diversity, which allows populations to adapt to potential environmental changes. This can be achieved by calculating how many offspring every individual should contribute to the next generation to minimise global coancestry. However, an undesired consequence of this strategy is that it maintains deleterious mutations, compromising the viability of the population. In order to avoid this, optimal contributions could be combined with inbred matings, to expose and eliminate recessive deleterious mutations by natural selection in a process known as purging. Although some populations that have undergone purging experienced reduced inbreeding depression, this effect is not consistent across species. Whether purging by inbred matings is efficient in conservation programmes depends on the balance between the loss of diversity, the initial decrease in fitness and the reduction in mutational load. Here we perform computer simulations to determine whether managing a population by combining optimal contributions with inbred matings improves its long-term viability while keeping reasonable levels of diversity. We compare the management based on genealogical information with management based on molecular data to calculate coancestries. In the scenarios analysed, inbred matings never led to higher fitness and usually maintained lower diversity than random or minimum coancestry matings. Replacing genealogical with molecular coancestry can maintain a larger genetic diversity but can also lead to a lower fitness. Our results are strongly dependent on the mutational model assumed for the trait under selection, the population size during management and the reproductive rate.
\end{abstract}

Heredity (2013) 110, 530-537; doi:10.1038/hdy.2012.119; published online 16 January 2013

Keywords: genetic diversity; inbreeding depression; purging; management; molecular coancestry

\section{INTRODUCTION}

The aim of conservation programmes is to maximise the survival probability of the population of interest (Frankham et al., 2002). That is best achieved by maintaining the largest possible genetic diversity, while also controlling the increase in inbreeding. Loss of genetic diversity is a major issue in conservation and evolutionary biology, as natural selection acts directly upon it and allows populations to adapt to changes in their environment. At the same time, populations in conservation programmes are usually small, or may have undergone bottlenecks, and thus, are at a risk of high rates of inbreeding. This can have a negative effect on their fitness, as it has been shown in many species which suffer inbreeding depression that can lead them to extinction (Frankham, 1995; Saccheri et al., 1998).

The best strategy to maintain the largest genetic diversity and to minimise the increase in inbreeding in a single population is using optimal contributions, that is, the number of offspring that each individual should contribute to the next generation to minimise global coancestry (Meuwissen, 1997; Grundy et al., 1998). In the optimisation of such contributions, genealogical coancestries can be used if available. Alternatively, we can use marker data to calculate molecular coancestries, which when based on a large number of single-nucleotide polymorphisms (SNPs) has proved to be more efficient for maintaining diversity than using genealogical information (de Cara et al., 2011).
However, by maintining global genetic diversity, we may be keeping mutations that are deleterious, thereby reducing the mean fitness of the populations. A strategy to avoid this problem is to combine optimal contributions with inbred matings, in order to expose deleterious recessive mutations, which will then be eliminated by natural selection in a process known as purging. Purging by inbred matings has been shown in some species to reduce the magnitude of inbreeding depression (Templeton and Read, 1983; Keller and Waller, 2002; Swindell and Bouzat, 2006; Leberg and Firmin, 2008). However, in populations of small census size inbred matings increase their extinction risk (Hedrick, 1994; Wang et al., 1999; Wang, 2000). Thus, purging by systematic inbreeding is still highly controversial in conservation programmes.

Simulation results (Wang et al., 1999; Wang, 2000) and single locus analysis (Glemin, 2003) have shown that the efficiency of purging by inbred matings is larger than purging by drift. The simulation studies (Wang et al., 1999; Wang, 2000) also showed that the efficiency of purging was highly dependent on the distribution of mutational effects of the trait under selection and on the census size of the population. Furthermore, demographic parameters like population size or reproductive rate, have been shown to be critical for populations to accumulate deleterious mutations that can lead to extinction (Lynch et al., 1995). These analyses did not explicitly

${ }^{1}$ Departamento de Mejora Genética Animal, INIA, Madrid, Spain and ²Departamento Producción Animal, E.T.S.I. Agrónomos, Universidad Politécnica de Madrid, Madrid, Spain Correspondence: Dr Ángeles de Cara, Departamento de Mejora Genética Animal, INIA (Instituto Nacional de Investigación y Tecnología Agraria y Alimentaria), Ctra. de La Coruña km. 7.5, Madrid 28040, Spain.

E-mail: rodriguez.angeles@inia.es

Received 12 July 2012; revised 10 December 2012; accepted 11 December 2012; published online 16 January 2013 
manage the populations in question, but allowed natural selection to act upon a selected trait.

Here, we analyse the effect of combining optimal contributions with inbred matings on the genetic diversity and fitness maintained in managed populations. We consider a fitness-related trait, controlled by a large number of loci and two opposite mutational models. We also study the effect of demography on management, by comparing different population sizes and reproductive rates. In order to perform the management, we use genealogical or molecular information.

\section{MATERIALS AND METHODS}

We ran stochastic simulations of diploid dioecious individuals with sexual reproduction under two different mutational scenarios. All simulations were performed in Fortran 90 and the code is available upon request. We firstly set up an ancestral population at mutation-selection-drift equilibrium. Consequently, linkage disequilibrium was generated between neutral, marker and selected loci. Different lines from this ancestral population were then used for management purposes.

\section{Generation of the ancestral population in mutation-selection-drift equilibrium}

We generated one population of $N=1000$ individuals with equal number of males and females. We assumed that individuals had 20 chromosomes of 1 Morgan each, and that fitness was controlled by a large number of selected loci, either 2000 or 20000 per genome, evenly distributed across chromosomes (that is, 100 or 1000 per chromosome, respectively). Fitness was multiplicative across these selected loci, and each selected locus $i$ had fitness $1,1-h_{i} s_{i}$ and $1-s_{i}$ for $A A, a A$ and aa genotypes, respectively. Parameters $s_{i}$ and $h_{i}$ are the selection and dominance coefficients at locus $i$, respectively.

The genome also included 2000 neutral biallelic loci and 1000 marker biallelic loci per chromosome, which were also evenly distributed. Marker loci were used for managing the population using molecular coancestry while neutral loci were used to measure diversity. All loci were initially fixed at the $A$ allele. Selected loci could mutate forward to the a allele, but backward mutation at selected loci was neglected. In order to obtain reasonable levels of diversity at neutral and marker loci, mutation from $A$ to $a$ and backward from $a$ to $A$ was allowed at these loci.

For the selected loci, we used two mutational models which assume different mutation rates and distributions of mutational effects, based on contrasting results from the literature. The first one is based on the results obtained by (Mukai et al., 1972) and is what we called the 'Mukai' scenario, where mutations are assumed to be numerous and of small effect. The second one is based on more recent results (Caballero and Keightley, 1994; Garcia-Dorado and Caballero, 2000), and is what we called the 'CGD' scenario, where mutations are considerably less frequent but of larger effect. Every generation there were on average $2 \lambda N$ deleterious mutations in the population, drawn from a Poisson distribution, which were distributed across all selected loci and individuals (that is, $\lambda$ is the mutation rate per haploid genome and generation). That is, deleterious mutations are distributed every generation by choosing random individuals, and within each of them, a random position in their genome, which is not already carrying a deleterious allele mutates. Mutations at selected loci had mean dominance coefficient $\bar{h}$ and mean selection coefficient $\bar{s}$. The selection coefficients were sampled from a Gamma distribution with shape parameter $\beta$ and mean $\bar{s}$. The dominance coefficient of each mutation was obtained from a uniform distribution between 0 and $\exp (-k s)$, where $k$ is a constant that gives the required $\bar{h}$, as proposed by Caballero and Keightley (1994). The parameters used for the Mukai scenario were $\lambda=0.5, \bar{s}=0.05, \bar{h}=0.35, \beta=1$, and for the CGD scenario were $\lambda=0.03, \bar{s}=0.264, \bar{h}=0.20, \beta=2.3$. The distribution of mutational effects for both scenarios is shown in Figure 1.

The number of mutations at marker and neutral loci were drawn from a Poisson distribution with mean $2 \mu N$, where $\mu=2.5 \times 10^{-3} n_{M}$ for marker loci, $\mu=2.5 \times 10^{-3} n_{N}$ for neutral loci and $n_{M}$ and $n_{N}$ are the number of marker and neutral loci per haploid genome, respectively. Thus, we are assuming an

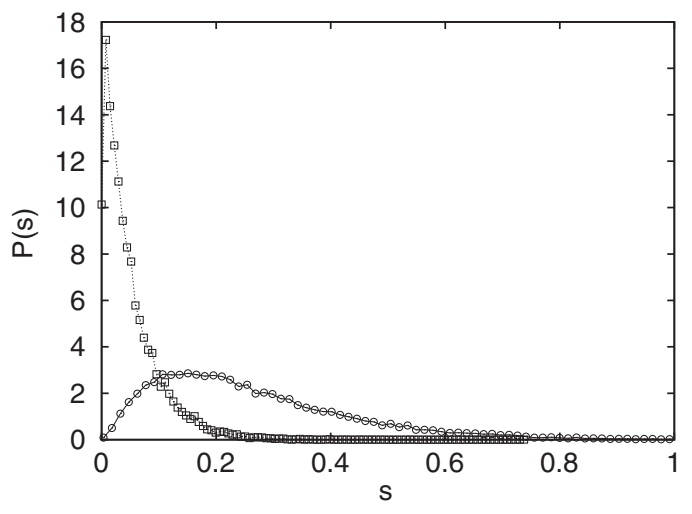

Figure 1 Distribution of mutational effects according to parameters used for the Mukai and the CGD scenarios. We show with squares the distribution of mutational effects for the Mukai scenario with $\beta=1$ and $\bar{s}=0.05$, and with circles, the distribution for the CGD scenario with $\beta=2.3$ and $\bar{s}=0.264$.

average mutation rate of $2.5 \times 10^{-3}$ per locus, both for marker and neutral loci.

The order of events was as followed: every generation we calculated the number of mutations in the population and distributed them across loci and individuals. Then we drew at random one male and one female and mated them. Gametes were produced with recombination, such that a number of crossovers was drawn from a Poisson distribution with mean 1 per Morgan. The fitness of their offspring was then calculated. As fitness is in the interval $(0,1)$, we compared the offspring fitness with a random number in such interval, and if the latter was smaller than fitness, this offspring survived, otherwise it died. This mating process was repeated with replacement by choosing a random male and a random female as many times as needed to produce a new population with $N$ offspring at reproduction age (with equal number 500 of males and females).

We iterated this scheme of mutation-reproduction-selection as many generations as needed to reach mutation-selection-drift equilibrium by measuring the mean heterozygosity of the population (usually, about 10000 discrete generations).

We stored the mean heterozygosity, mean fitness of the population, the average frequency of the deleterious allele $a$ and the inbreeding load during this period. The inbreeding load $\delta=\sum_{i} 2 p_{i} q_{i}\left(1-h_{i} s_{i}-\frac{\left(2-s_{i}\right)}{2}\right)$ (Morton and Crow, 1956) measures the rate of decay in log fitness due to inbreeding only. For instance, fitness at time $t$ is $w_{t}=w_{0} \exp (-\delta F) \approx w_{0}(1-\delta F)$, where $F$ is the inbreeding coefficient, in the absence of selection, as shown in Falconer and Mackay (1996).

\section{Management}

In order to test whether purging by inbred matings was a useful option we managed replicate populations of $N_{p}=100$ individuals (50 females and 50 males), except for some simulations of $N_{p}=10$ individuals ( 5 females and 5 males), during 10 generations. $N_{p}$ is the population size during management, in contrast with $N$, which is the ancestral population size. These $N_{p}$ individuals were drawn randomly without replacement from the larger ancestral population at mutation-selection-drift equilibrium. In what follows, we will call $t=0$ to the first generation where management was applied.

Every management generation consisted of three steps: firstly, we calculated how many contributions each individual should leave; secondly, matings were arranged between contributing individuals; thirdly, each mating produces offspring to form the next generation.

1. Contributions. We calculated which individuals should contribute to the next generation and how many offspring they should leave, by minimising the global coancestry weighted by those contributions, that is,

$$
\sum_{i=1}^{N_{p}} \sum_{j=1}^{N_{p}} \frac{c_{i} c_{j} f_{i j}}{T^{2}}
$$


where $c_{i}$ is the contribution of individual $i$ (that is, the number of offspring that $i$ leaves to the next generation) and $f_{i j}$ is the coancestry between individuals $i$ and $j . T$ is the sum of the contributions $\sum_{i} c_{i}$ (Fernandez et al., 2003; Fernandez et al., 2005), which is set at $2 N_{p}$, and thus, the total number of intended contributions is constant during the management time. We also imposed the restriction that the sum of contributions of the females is equal to the sum of contributions from the males. This optimisation minimises the expected average coancestry on the offspring. We also compared contributions of minimum coancestry with contributions at random, that is, with random c's, which satisfy all the other constraints.

2. Matings. Those individuals whose contributions were greater than zero were mated to produce offspring. We simulated three mating scenarios: (a) matings of minimum coancestry, (b) matings of maximum coancestry or (c) matings at random. We did this by calculating the average pairwise coancestry between couples that a combination of matings would yield. Matings of maximum (minimum) coancestry were achieved by searching for the combination of matings that yielded the maximum (minimum) average pairwise coancestry. That is, we maximised (minimised) $\sum_{a, b} t_{a, b} f_{a, b}$, where $f_{a, b}$ is the coancestry between contributing male $a$ and female $b$, and $t_{a, b}$ is one if $a$ and $b$ mate and zero otherwise. Note that each individual can participate in as many matings as required to produce its optimal number of offspring. Both the calculation of the optimal contributions and the arrangement of matings were done using 'simulated annealing' algorithms (see Supplementary Information)

The coancestry between individuals, $f_{i j}$, which is needed to calculate the contributions and to arrange matings, was calculated either with genealogies or with molecular information from the marker loci. When we managed using genealogies we assumed that the population is composed of unrelated individuals at $t=0$. When we managed the population using molecular information, we only used marker loci that were segregating every generation to calculate molecular coancestry. Molecular coancestry was calculated applying Malecot (1948) definition but referring to identity in state instead of identity by descent (as done in Toro et al., 2002).

3. Offspring. During management there was no mutation, but there was still selection. The fitness of the offspring of each mating pair was calculated and compared with a random number, and if the random number was smaller than the fitness of the zygote, this offspring was kept. This process was repeated up to $K$ times, to simulate a limited reproductive rate (as in a limited litter size). It is possible that none of the $K$ attempts of a mating pair succeeded in leaving offspring if they all had very low fitness. As each individual participates in as many mating pairs as contributions is leaving, its reproductive rate would be the sum of $K$ 's over each pair in which they are involved. If a mating pair does not leave offspring, the population size decreases. However, the population size can recover in the next generation, as during the calculation of contributions, the total number of contributions remains constant through management (equal to $N_{p}$ ).

We show here results of fitness measured after selection and observed heterozygosity measured on neutral loci that are segregating at $t=0$, averaged over individuals and replicates. We have also measured expected heterozygosity, observed heterozygosity averaged over all neutral loci, and genealogical inbreeding.

\section{RESULTS}

At $t=0$, the populations with 1000 selected loci per chromosome had a very low polymorphism (over $75 \%$ of all loci were fixed, and on average, 76 and $98 \%$ of the selected loci were fixed in the Mukai and CGD scenarios, respectively). A summary of mean values for the ancestral population with 1000 selected loci for both mutational scenarios can be found in Table 1 .

The frequency of the deleterious allele $a$ averaged over all selected loci at $t=0$ was $4.98 \times 10^{-3}$ and $6.74 \times 10^{-5}$ for 1000 selected loci for the Mukai and CGD mutational scenarios, respectively. The values of mean fitness, mean number of loci per individual carrying
Table 1 Mean fitness $(\bar{w})$, mean number of loci carrying a deleterious allele a per individual $\left(\bar{n}_{a}\right)$, mean number of segregating loci averaged over individuals $\left(\bar{n}_{\text {seg }}\right)$, average inbreeding load $(\bar{\delta})$ averaged over the last 1000 generations before reaching equilibrium, and average $\bar{r}^{2}$ over the first five positions away from each locus in the ancestral populations in the Mukai and the CGD scenarios with 1000 selected loci, 1000 markers and 2000 neutral loci per chromosome

\begin{tabular}{lcc}
\hline & Mukai & CGD \\
\hline $\bar{w}$ & $0.47(0.05)$ & $0.95(0.05)$ \\
$\bar{n}_{a}$ & $221.70(11.25)$ & $2.45(1.53)$ \\
$\bar{n}_{\text {seg }}$ & $559.37(33.37)$ & $2513.55(60.02)$ \\
$\bar{\delta}$ & $0.99(0.04)$ & $0.25(0.03)$ \\
$\bar{r}^{2}$ & 0.41 & 0.32 \\
\hline
\end{tabular}

s.d. for each mean are given in brackets.

deleterious alleles and other averages at $t=0$ for both mutational scenarios are given in Table 1. We also include in that Table the value of linkage disequilibrium measured as $r^{2}=\sum_{i=1}^{2} \sum_{j=1}^{2} \frac{D_{i j}^{2}}{\left(1-p_{i}\right)\left(1-p_{j}\right)}$, where $D_{i j}$ is the linkage disequilibrium between loci $i$ and $j$, and $p_{i}$ and $p_{j}$ are allelic frequencies, measured from the adjacent position to five positions away and averaged over all loci.

As mentioned above, we measured genetic diversity during management via expected and observed heterozygosity, averaged over neutral loci or over all loci (selected, neutral and marker). As the temporal evolution during management of these variables of diversity were similar, we only show here observed heterozygosity in neutral loci that are segregating at $t=0$, which for simplicity, we will refer to in the following as heterozygosity. We will also refer to random management as the combination of random contributions with random matings, as explained in the Management subsection. We averaged the results over 100 replicates, none of which went extinct for the combination of parameters and scenarios here shown. The temporal evolution of the mean fitness of the population and the observed heterozygosity in neutral loci initially segregating are shown in Figures $2-5$, scaled to the initial values at $t=0$. Results for scenarios with 100 selected loci per chromosome can be found in Supplementary Information.

\section{Molecular or genealogical-based management}

Managing the population using molecular coancestry maintained generally a higher heterozygosity than managing the population using genealogical coancestry. This is particularly the case when $K$ is large (right panels of Figures 2 and 3), for both mutational scenarios. Under the CGD mutational model, the population has been purged of most deleterious mutations before management, and we can maintain more heterozygosity using molecular coancestry for large $K$ without impacting fitness. We can also see that when marker loci are tightly linked to neighbouring selected loci, as in the case of 1000 selected loci using molecular coancestry maintains more heterozygosity than genealogical coancestry (results for 100 selected loci are shown in Supplementary Information). The results for large $K$ under the CGD scenario are similar to a neutral scenario, in line with the results obtained by de Cara et al. (2011). In that study, in the absence of selection, more heterozygosity is maintained using molecular than genealogical information when the number of markers is large enough.

The observation that using molecular coancestry maintains more heterozygosity in the CGD scenario without hardly any impact on 


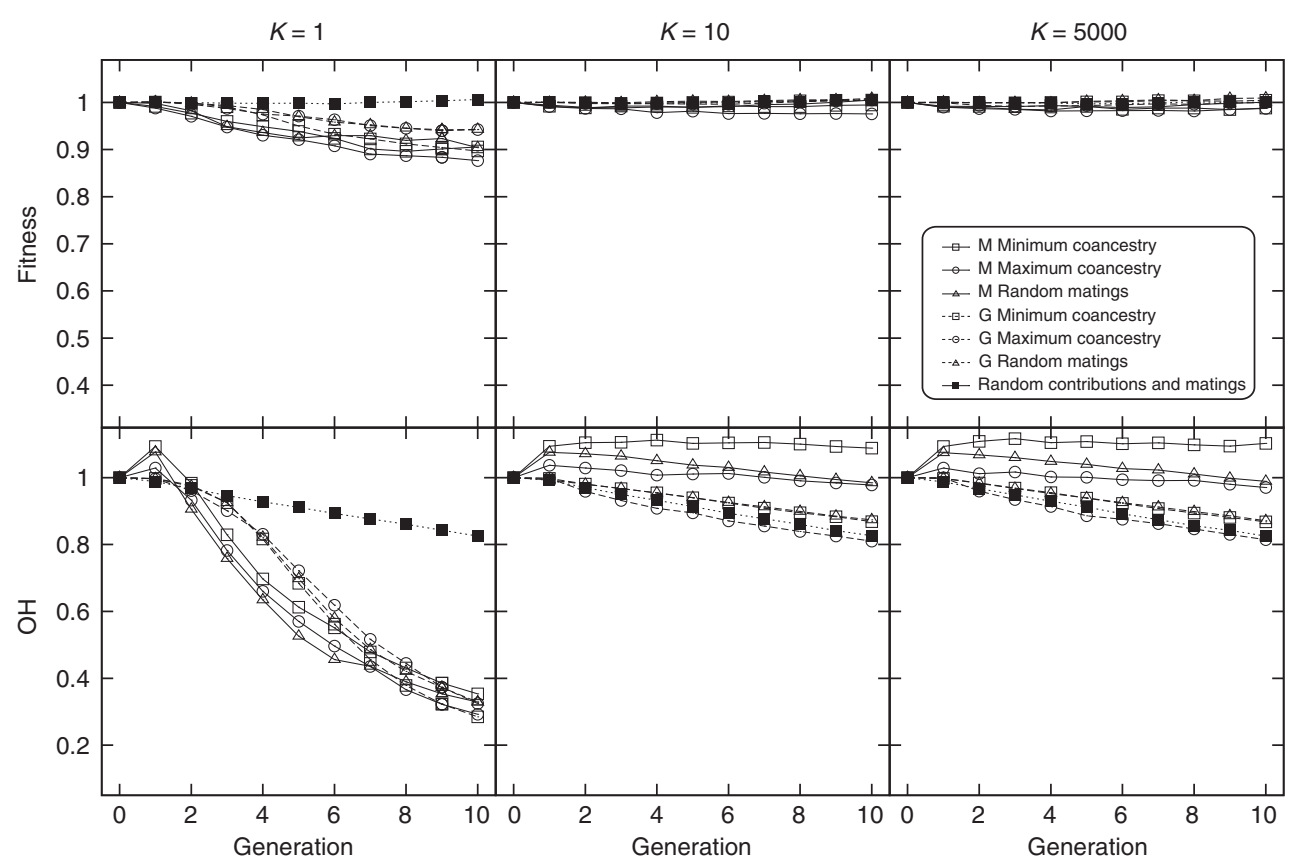

Figure 2 Observed heterozygosity averaged over all neutral loci that are initially polymorphic, and mean fitness plotted against management generations, under the CGD scenario with 1000 selected loci per chromosome, using molecular (M) or genealogical (G) coancestry and different mating strategies. The results show averages over 100 replicates, and the maximum s.d. in mean fitness is 0.005 at $t=0$ and 0.03 at $t=10$, and in observed heterozygosity at neutral sites initially segregating is 0.001 at $t=0$ and 0.007 at $t=10$.

fitness contrasts with the results obtained for the Mukai mutational scenario (Figure 3). For that scenario, the population has a much larger initial inbreeding load and considerably lower fitness (see Table 1), and there are many more deleterious mutations segregating. Thus, if in that scenario management maintains more overall heterozygosity, this leads to maintaining more deleterious mutations, and thus lower fitness, as can be clearly seen in the rightmost panels in Figure 3.

The reason for the association between an increase in heterozygosity and a decrease in fitness in the Mukai scenario is the tight linkage between markers and neutral and selected loci. Thus, increasing heterozygosity at marker loci, used to compute molecular coancestry, increases heterozygosity at linked neutral or selected loci (de Cara et al., 2011). Thus, if more heterozygosity at selected loci is maintained, including deleterious alleles, there will be a decrease in fitness. This lower fitness obtained when managing with molecular information under the Mukai scenario held during the ten generations considered, and the difference between using molecular or genealogical coancestry seems to be still increasing at the end of the management period.

\section{Demographic effects: limited $K$}

Under the CGD mutational model (Figure 2) the performance for $K=10$ is similar to that observed with unlimited $K$, where more heterozygosity and similar fitness is maintained using molecular than genealogical information. However, very different results are obtained for $K=10$ in the Mukai mutational scenario (Figure 3) and for $K=1$ in both mutational scenarios (left panels in Figures 2 and 3), where using genealogical information maintained more heterozygosity and led to higher fitness than using molecular information.

In the Mukai scenario and for $K=10$ (Figure 3), there was a small reduction in census size after selection, which would hardly justify such decrease in fitness compared with the case of $K=5000$. This decay is mostly due to the decrease in the number of individuals that actually contribute to the next generation. This number is always 100 , and thus equal to $N_{p}$, for $K=5000$ and as low as 53 at $t=10$ for $K=10$ when using genealogical information. This effect is more pronounced when using molecular information. For instance, for $K=10$ the number of individuals contributing at $t=10$ was slightly over $50 \%$ of those contributing with $K=5000$ when using genealogical informating, while this number is, on average, below $16 \%$ when using molecular information.

The effect of selection was weaker as we decreased $K$ to the extent that for $K=1$, for both mutational scenarios, managing at random (random contributions and random matings, though limited $K$ ) maintained the largest fitness and heterozygosity. Genealogical inbreeding was about 10 times larger at $t=10$ in populations managed with genealogical information than in populations where contributions and matings were performed at random (results not shown). The number of individuals contributing to matings in a population managed using molecular or genealogical information and matings of maximum or minimum coancestry was considerably lower than when the population was managed at random (for instance, in the Mukai scenario, for $K=1$ and 1000 selected loci, at $t=10$, managing at random allowed an average of $66 \%$ individuals to contribute, while managing with genealogical or molecular information only $5 \%$ and $10 \%$ of individuals contribute, respectively). Thus, natural selection could act upon those contributing individuals and maintain the fittest.

\section{Mating schemes}

In Figures 2 and 3, we can see how the effect of arranging matings by minimum or maximum coancestry or at random is small. Thus, once contributions have been decided, how matings are performed has a 


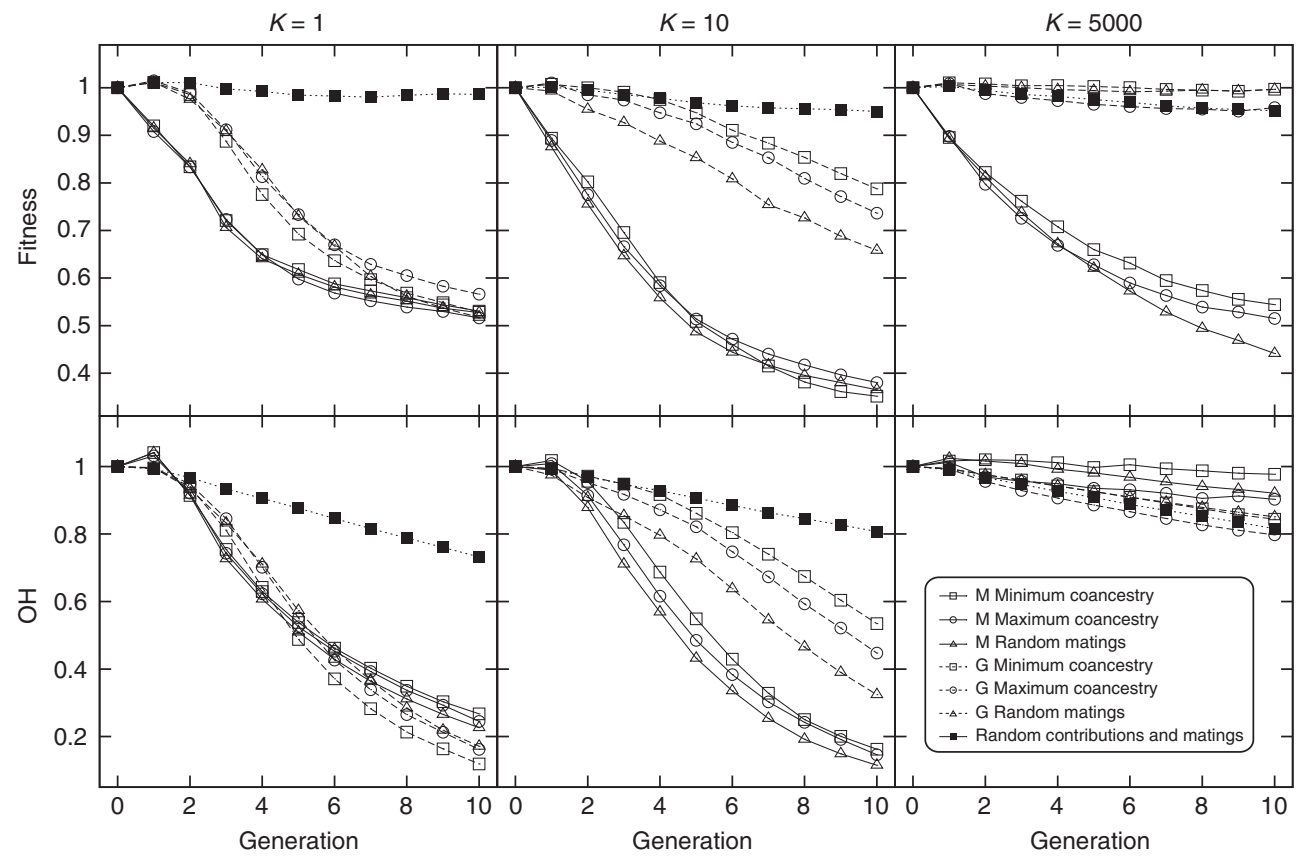

Figure 3 Observed heterozygosity averaged over all neutral loci that are initially polymorphic, and mean fitness plotted against management generations, under the Mukai scenario with 1000 selected loci per chromosome, using molecular (M) or genealogical (G) coancestry and different mating strategies. The results show averages over 100 replicates, and the maximum s.d. in mean fitness is 0.004 at $t=0$ and 0.07 at $t=10$, and in observed heterozygosity at neutral sites initially segregating is 0.001 at $t=0$ and 0.004 at $t=10$.

small impact on fitness and heterozygosity in the 10 generations of management studied. Furthermore, for $N_{p}=100$ there is no scenario, in which more fitness is maintained by performing matings of maximum coancestry than by performing matings of minimum coancestry. In the CGD scenario, where most deleterious mutations have been eliminated before management and fitness is initially very large, performing matings of maximum coancestry maintained less heterozygosity than performing matings at random (Figure 2), although the differences were very small. The differences in fitness in that mutational scenario are extremely small. For the Mukai scenario (Figure 3), where there are many more deleterious mutations segregating and fitness is initially much lower than for the CGD mutational scenario, performing matings of maximum coancestry sometimes maintains a higher fitness than performing matings at random. Sometimes matings of maximum coancestry under this mutational scenario maintain more heterozygosity than matings at random. This happens because of the higher opportunities of purging provided by maximum coancestry matings, which leads to this strategy to allow more individuals to leave offspring. Although the probability of transmitting genetic information in one generation depends on the contributions and these are equal for both mating schemes, in the long-term forcing inbreeding can maintain more heterozygosity as drift is avoided when dealing with homozygous individuals. Overall, for a population size of $N_{p}=100$, the method that maintains the largest fitness and heterozygosity is to arrange matings by minimum coancestry.

\section{Small population size}

We have seen how for a large $N_{p}=100$ and very small reproductive rate $K$, managing at random maintained more fitness and heterozygosity. In order to see the generality of these results, we have performed simulations for smaller population sizes during management, and we show here the extreme case of $N_{p}=10$, with equal number of males and females at the beginning of management. Most noticeably, using molecular information maintained more heterozygosity in both mutational scenarios, for both large or limited reproductive rate $K$, and managing at random is not the best strategy for this population size during management.

Under the Mukai scenario (right panels in Figure 4), and for large reproductive rate $K$, the decay in fitness for $N_{p}=100$ is much faster than for $N_{p}=100$, particularly using genealogical information. Managing using random contributions and performing matings at random maintains the least fitness and heterozygosity than with any other sort of management. For this mutational scenario and $K=10$ (right panels of Figure 5), the difference between management strategies are smaller compared with large $K$, more fitness is maintained with genealogical information and random management maintains the least heterozygosity.

As linkage disequilibrium is considerably higher at larger distances in the genome for smaller population sizes than for larger population sizes, we could have expected molecular management to maintain even more heterozygosity than for larger population sizes. However, selection is less efficient for small population sizes, and while more heterozygosity is maintained with molecular management, fitness decays more quickly than for large population size (see right panels of Figures 3 and 4), and this leads to the difference between molecular and genealogical management to be small.

For the CGD scenario (left panels of Figures 4 and 5), more heterozygosity is maintained using molecular information, and is lower managing at random. In this mutational scenario, the differences between methods in maintaining fitness are small, and it seems that for populations with large reproductive rate $(K=5000)$ fitness can experience a slight recovery. This would indicate that purging has been efficient in this mutational scenario. 


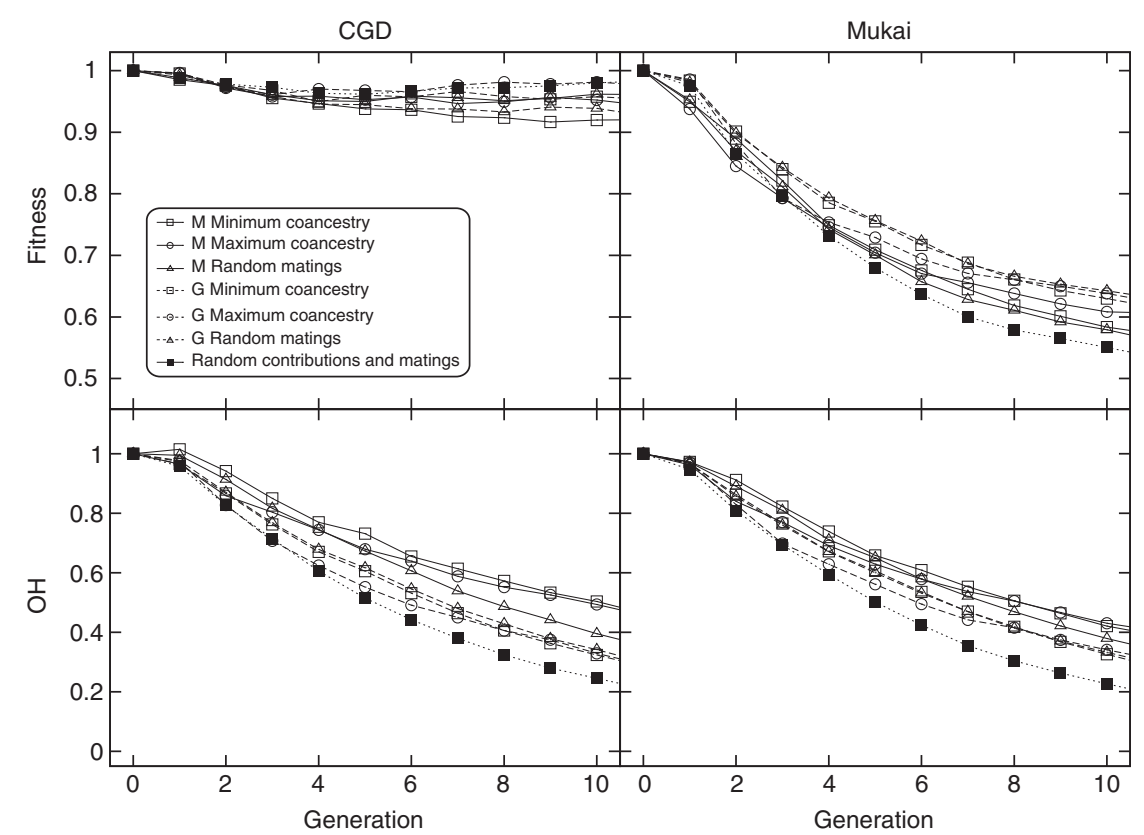

Figure 4 Management for a population of $N_{p}=10$ at $t=0$ and $K=5000$ under the CGD and Mukai scenarios with 1000 selected loci. Observed heterozygosity averaged over all neutral loci that are initially polymorphic and mean fitness, plotted against management generations using molecular (M) or genealogical (G) coancestry and different mating strategies. The results show averages over 100 replicates, and the maximum standard deviation in mean fitness is 0.05 at $t=0$ and 0.07 at $t=10$, and in observed heterozygosity at neutral sites initially segregating is 0.001 at $t=0$ and 0.004 at $t=10$.

\section{DISCUSSION}

In this study, we have combined inbred matings to expose deleterious mutations with optimal contributions to maximise heterozygosity in the management of populations in conservation programmes. The aim was to obtain an eventual increase in fitness, which would mean that purging has been efficient, but overall, we have not seen such increase in the 10 generations of management here studied. Furthermore, not only we have not seen an increase in fitness, but we have shown here that performing inbred matings leads to a lower fitness than other mating schemes, at least for the time period and population sizes here considered. We have also shown that molecular information can maintain more heterozygosity, but this can lead to a reduced fitness. Finally, the interaction of the different factors, such as genetic architecture assumed, both in terms of the mutational model and the number of selected loci, and the reproductive rate have a strong impact to determine the best management strategy.

Once contributions have been decided, the strategy followed to mate individuals does not have much of an impact in maintaining heterozygosity and improving fitness. Between family selection is largely reduced, and selection is mostly within family. We expected that performing inbred matings would create more homozygous offspring and selection would be most effective. However, this effect does not seem to compensate the loss in fitness due to inbreeding depression. Thus, the combination of manipulating contributions and manipulating matings does not largely improve the results in terms of maintaining diversity and fitness that would be obtained by only manipulating contributions. Alternatively, we could apply strategies to increase within family selection, by applying artificial selection (as already performed by Fernandez and Caballero, 2001b), but this will depend on the reproductive rate of the species under conservation.

Slightly larger variability and fitness may be maintained if we applied strategies that distinguish between those individuals that contribute the most to inbreeding (Garcia-Cortes et al., 2010), or ancestral inbreeding from recent inbreeding (Ballou, 1997). The harmful effects of inbreeding are due to recent inbreeding, as the population has overcome ancestral inbreeding (Swindell and Bouzat, 2006). Possibly, we could maximise heterozygosity based on ancestral inbreeding and perform inbred matings based on recent inbreeding.

In our study, we have assumed a large number of marker loci, in line with the large single-nucleotide polymorphism panels that are now available. These panels are available mainly for species of economic interest and we expect that there will be soon plenty of molecular data for other species, like wild species in conservation programmes. We were motivated by earlier results (de Cara et al., 2011), in which more heterozygosity could be maintained using molecular information than using genealogical information under a neutral scenario. Our previous study encouraged the use of molecular information based on these large panels in the management of populations, in the absence of a trait under selection. The results here shown indicate that molecular information has to be used with caution, as it can lead to maintaining deleterious mutations tightly linked to the markers used in the calculation of molecular coancestry.

The initial fitness of the population highly depends on the genetic architecture of the trait under selection. If mutations are few and of large deleterious effects (CGD scenario), then the impact of management on fitness is much more reduced than if there are many mildly deleterious mutations (Mukai scenario). Thus, the mutational model is crucial for the results here obtained. There is no consensus on what is the most plausible mutational model (Halligan and Keightley, 2009; Lopez-Fanjul and Garcia-Dorado, 2010). The parameters derived by Mukai and those who followed his experiments (Mukai, 1964; Mukai et al., 1972; Ohnishi, 1977a,b) have been commonly used in many simulation studies, while there is growing evidence for other distributions of mutational effects (Fernandez and Lopez-Fanjul, 1996; Shaw et al., 2000; Caballero et al., 2002). Recently, Halligan and Keightley (2009) have concluded that the distribution of mutational effects is most likely to be somewhere in between our Mukai and CGD mutational scenarios. The estimation of the genomic 


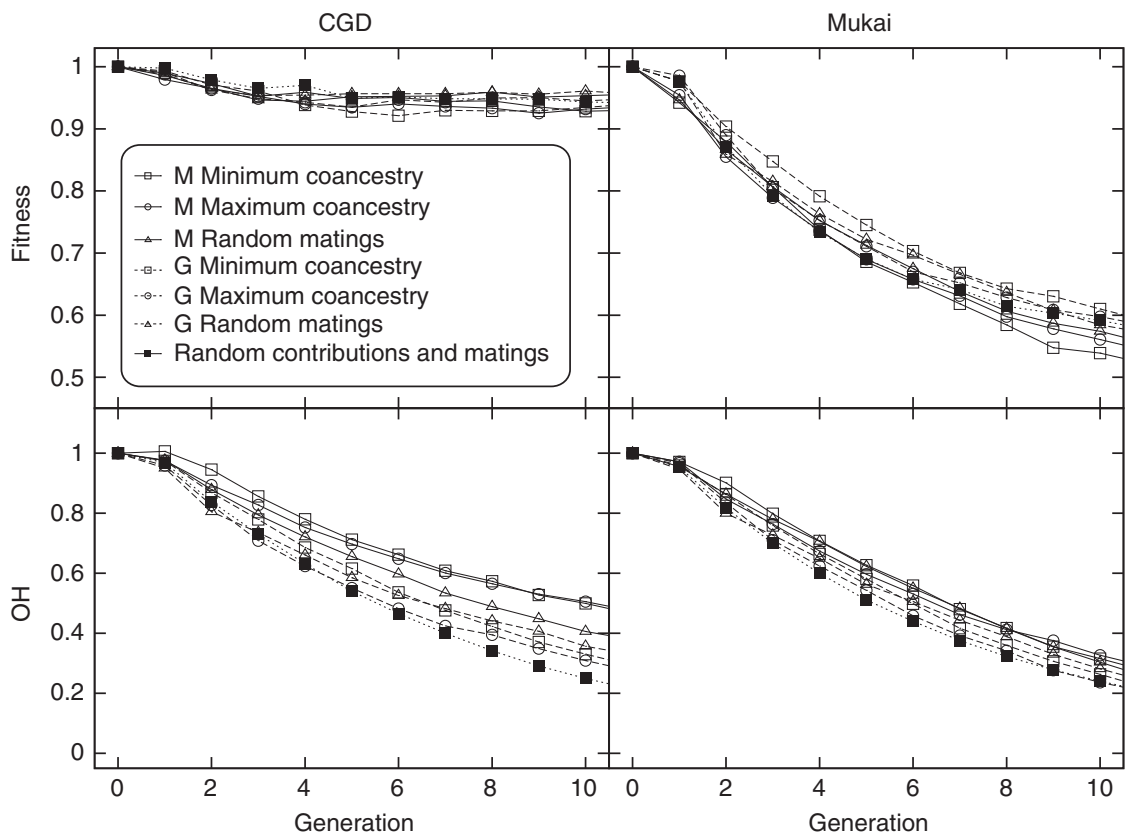

Figure 5 Management for a population of $N_{p}=10$ at $t=0$ and $K=10$ under the CGD and Mukai scenarios with 1000 selected loci. Observed heterozygosity averaged over all neutral loci that are initially polymorphic and mean fitness, plotted against management generations using molecular (M) or genealogical (G) coancestry and different mating strategies. The results show averages over 100 replicates, and the maximum s.d. in mean fitness is 0.05 at $t=0$ and 0.08 at $t=10$, and in observed heterozygosity at neutral sites initially segregating is 0.001 at $t=0$ and 0.004 at $t=10$.

mutation rate and the average mutational effect is difficult in mutation-accumulation experiments, as both parameters are strongly confounded. Recent research based on whole-genome sequencing points towards a complex distribution of mutational effects (EyreWalker and Keightley, 2007), with average mean effects varying widely between species. We have compared here two extreme distributions of mutational effects and believe that intermediate distributions will give results somewhere between the two here obtained.

The mutational model assumed led to an initial population prior to management with a very large fitness (CGD scenario) or a reduced fitness (Mukai scenario). This had a combined impact when the reproductive rate $(K)$ was limited. If the population has a high reproductive rate, then selection can act on those individuals who contribute. When the reproductive rate is limited, then selection is less efficient in eliminating deleterious mutations. This leads to a threshold where managing at random maintains more heterozygosity and fitness than managing minimising coancestry. The limit when managing at random maintains more fitness and heterozygosity than managing minimising coancestry depends on the population size during management, the reproductive rate and the initial fitness of the population (which depends on the mutational model assumed). For instance, for a population of $N_{p}=100$ and 1000 selected loci, under the Mukai mutational scenario for $K=10$ managing at random maintains more fitness and diversity, while for such $N_{p}$ and selected loci, this does not occur until $K=1$ for the CGD mutational scenario. For a population of $N_{p}=10$ and 1000 selected loci, this limit is lower. Thus, demographic effects like reproductive rate and census size can thus have a larger impact on heterozygosity than managing the population, in agreement with the results found by Ivy and Lacy (2012). However, while we have shown cases when managing at random is better than managing using contributions of minimum coancestry, this result is not universal, and it depends on population size, reproductive rate (or litter size) and population fitness. In practice, if conservation managers have information on the fitness of each individual, management could be constrained not to decline the mean fitness of the population, if possible, as performed in the analysis of Fernandez and Caballero (2001b).

The results here obtained for population fitness for both mutational scenarios are in line with those obtained by Fernandez and Caballero (2001a,b), who only used genealogical information. Our study is the first one to show the differences between using molecular or genealogical information in maintaining fitness and heterozygosity, as well as forcing inbred matings in an attempt to expose deleterious mutations. Garcia-Dorado (2012) has recently derived formulae that approximate the results for viability during management with equalfamily contributions, as performed in the study by Fernandez and Caballero (2001a). Those approximations work well compared with simulations for a mutational scenario closer to CGD. Interestingly, Garcia-Dorado (2012) has found some scenarios where there is a fitness rebound about $10-20$ generations of management, for a population size of 10 and one selected locus. We have obtained similar results for such population size and many selected loci under the CGD scenario (results not shown). It would be interesting then to compare the analytical approximations, which assume independent selected loci, with optimal contributions for a range of population sizes to further understand the complex interaction between census size, reproductive rate, selection and linkage. Long-term vs short-term benefits of management could then be analysed considering mutation during management. Furthermore, we could consider what would happen when our managed populations are reintroduced in the wild, by looking at the fitness of panmictic populations, which could determine to which extent purging of deleterious mutations has occurred.

In summary, we have found that once contributions are decided, although the use of inbred matings may favour selection to eliminate deleterious mutations, the efficiency of selection for purging is 
reduced and the initial loss in fitness due to increased inbreeding depression is not recovered during the management period. Therefore, in conservation programmes, managing contributions is more effective than managing matings to maintain fitness and diversity, when there is initially a reasonable balance between the diversity and the genetic load. We have also shown that molecular information is very efficient to maintain heterozygosity, but also maintains deleterious mutations with harmful consequences for the population fitness. Lastly, there is a strong interaction between the mutational model and demographic parameters, like census size or reproductive rate, which determine which management strategy should be used.

\section{DATA ARCHIVING}

The simulated genotypes used for population management have been deposited in Dryad with provisional DOI doi:10.5601/dryad.bg40m. Four files are there included, one for each genetic architecture: for the Mukai and the CGD scenarios with 1000 and 100 selected loci per chromosome.

\section{CONFLICT OF INTEREST}

The authors declare no conflict of interest.

\section{ACKNOWLEDGEMENTS}

We are most grateful to Aurora Garcia-Dorado for comments on the work and on the manuscript. This work was funded by the Ministerio de Ciencia e Innovación (CGL2009-13278-C02-02, cofunded by FEDER).

Ballou JD (1997). Ancestral inbreeding only minimally affects inbreeding depression in mammalian populations. J Hered 88: 169-178.

Caballero A, Cusi E, Garcia C, Garcia-Dorado A (2002). Accumulation of deleterious mutations: additional Drosophila Melanogaster estimates and a simulation of the effects of selection. Evolution 56: 1150-1159.

Caballero A, Keightley P (1994). A pleiotropic nonadditive model of variation in quantitative traits. Genetics 138: 883-900

Cara MAR, Fernandez J, Toro MA, Villanueva B (2011). Using genome-wide information to minimize the loss of diversity in conservation programmes. J Anim Breed Genet $\mathbf{1 2 8}$ 456-464.

Eyre-Walker A, Keightley P (2007). The distribution of fitness effects of new mutations. Nat Rev Genet 8: 610-618.

Falconer DS, Mackay TFC (1996). Introduction to Quantitative Genetics. Longman: Essex, England.

Fernandez J, Caballero A (2001a). Accumulation of deleterious mutations and equalization of parental contributions in the conservation of genetic resources. Heredity $\mathbf{8 6}$ 480-488.

Fernandez J, Caballero A (2001b). A comparison of management strategies for conservation with regard to population fitness. Conserv Genet 2: 121-131.

Fernandez J, Lopez-Fanjul C (1996). Spontaneous mutational variances and covariances for fitness-related traits. Genetics 143: 829-837.

Fernandez J, Toro MA, Caballero A (2003). Fixed contributions designs vs. minimization of global coancestry to control inbreeding in small populations. Genetics 165: 885-894.
Fernandez J, Villanueva B, Pong-Wong R, Toro MA (2005). Efficiency of the use of pedigree and molecular marker information in conservation programs. Genetics 170 : $1313-1321$.

Frankham R (1995). Conservation genetics. Annu Rev Genet 29: 305-327.

Frankham R, Ballou JD, Briscoe DA (2002). Introduction to Conservation Genetics. Cambridge University Press: Cambridge, UK.

Garcia-Cortes LA, Martinez-Avila JC, Toro MA (2010). Fine decomposition of the inbreeding and the coancestry coefficients by using the tabular method. Conserv Genet 11: 1945-1952.

Garcia-Dorado A (2012). Understanding and predicting the fitness decline of shrunk populations: inbreeding, purging, mutation, and standard selection. Genetics 190: 1461-1476.

Garcia-Dorado A, Caballero A (2000). On the average degree of dominance of deleterious spontaneous mutations. Genetics 155: 1991-2001.

Glemin S (2003). How are deleterious mutations purged? Drift versus nonrandom mating. Evolution 57: 2678-2687.

Grundy B, Villanueva B, Wooliams JA (1998). Dynamic selection procedures for constrained inbreeding and their consequences for pedigree development. Genet Res 72: 159-168.

Halligan DL, Keightley PD (2009). Spontaneous mutation accumulation studies in evolutionary genetics. Ann Rev Ecol Evol Syst 40: 151-172.

Hedrick PW (1994). Purging inbreeding depression and the probability of extinction: Fullsib mating. Heredity 73: 363-372.

Ivy JA, Lacy RC (2012). A comparison of strategies for selecting breeding pairs to maximize genetic diversity retention in managed populations. J Hered 103: 186-196.

Keller LF, Waller DM (2002). Inbreeding effects in wild populations. Trends Ecol Evol 17: 230-241.

Leberg PL, Firmin BD (2008). Role of inbreeding depression and purging in captive breeding and restoration programmes. $\mathrm{Mol}$ Ecol 17: 334-343.

Lopez-Fanjul C, Garcia-Dorado A (2010). The fuel of evolution. Heredity 106: 535-536.

Lynch M, Conery J, Bürger R (1995). Mutation accumulation and the extinction of small populations. Am Nat 146: 489-518.

Malecot G (1948). Les Mathematiques de l'Heredite. Masson et Cie: Paris, France.

Meuwissen THE (1997). Maximizing the response of selection with a predefined rate of inbreeding. J Anim Sci 75: 934-940.

Morton NE, Crow JF (1956). An estimate of the mutational damage in man from data on consanguineous marrianges. Proc Natl Acad Sci USA 42: 855-863.

Mukai T (1964). The genetic structure of natural populations of Drosophila Melanogaster. I. Spontaneous mutation rate of polygenes controlling viability. Genetics 50: 1-19.

Mukai T, Chigusa SI, Mettler LE, Crow JF (1972). Mutation rate and dominance of genes affecting viability in Drosophila Melanogaster. Genetics 72: 333-355.

Ohnishi O (1977a). Spontaneous and ethyl methanesulfonate-induced mutations controlling viability in Drosophila Melanogaster. II. Homozygous effects of polygenic mutations. Genetics 87: 529-545.

Ohnishi O (1977b). Spontaneous and ethyl methanesulfonate-induced mutations controlling viability in Drosophila Melanogaster. III. Heterozygous effects of polygenic mutations. Genetics 87: 547-556.

Saccheri I, Kuusari M, Kankare M, Vikman P, Fortelius W, Hanski I (1998). Inbreeding and extinction in a butterfly metapopulation. Nature 392: 491-494.

Shaw FH, Geyer CJ, Shaw RG (2000). A comprehensive model of mutations affecting fitness and inferences for Arabidopsis Thaliana. Genetics 155: 369-378.

Swindell WR, Bouzat JL (2006). Ancestral inbreeding reduces the magnitude of inbreeding depression in Drosophila Melanogaster. Evolution 60: 762-767.

Templeton AR, Read B (1983). The elimination of inbreeding depression in a captive herd of Speke's gazelle. In: Schonewald-Cox CM, Chambers SM, MacBryde B, Thomas WL (eds). Genetics and Conservation. Benjamin/Cummings Publishing Company: Menlo Park, California, pp 241-261.

Toro MA, Barragan C, Ovilo C, Rodrigañez J, Rodriguez C, Silio L (2002). Estimation of coancestry in Iberian pigs using molecular markers. Cons Genet 3: 309-320.

Wang J (2000). Effects of population structures and selection strategies on the purging of inbreeding depression due to deleterious mutations. Genet Res 76: 75-86.

Wang J, Hill WG, Charlesworth D, Charlesworth B (1999). Dynamics of inbreeding depression due to deleterious mutations in small populations: mutation parameters and inbreeding rate. Genet Res 74: 165-178.

Supplementary Information accompanies the paper on Heredity website (http://www.nature.com/hdy) 